\title{
Antioxidants in the Prevention and Treatment of Diabetic Retinopathy - A Review
}

\author{
Sara Baptista da Silva ${ }^{1 *}$, João Paulo Costa², Manuela Estevez Pintado³, Domingos de Carvalho Ferreira1 and Bruno Sarmento ${ }^{1,4}$
}

${ }^{1}$ Department of Pharmaceutical Technology, Faculty of Pharmacy, University of Porto, Rua Aníbal Cunha 164, 4050-047 Porto, Portugal

${ }^{2}$ Laser Vision Correction Surgical Center, Avenida de França 256,7.4, 4050-276 Porto, Portugal

${ }^{3}$ Center of Biotechnology and Fine Chemistry, Biotechnology School of Portuguese Catholic University, Rua Dr. António Bernardino de Almeida $4200-072$ Porto, Portugal

${ }^{4}$ CICS, Department of Pharmaceutical Sciences, Instituto Superior de Ciências da Saúde - Norte, Rua Central de Gandra, 1317, 4585-116 Gandra, Portugal

\begin{abstract}
Diabetic retinopathy is a disease resulting from diabetic chronic hyperglycemia characterized by microvascular complications in the retina, where neuronal elements responsible for vision are located. It is the main cause of adult blindness in developed countries. Oxidative stress has been widely regarded as the key factor for the emergence of ocular disease and has been involved in increased vascular permeability, disruption of blood-retinal barrier, apoptotic loss of retinal capillary cells, microvascular abnormalities and retinal neovascularization. Dietary supplementation with antioxidants has been related with inhibition of diabetes-induced abnormalities of retinal metabolism, reduction of apoptosis and partial restoration of pericytes. Moreover, the use of topical antioxidants to treat or delaying oxidative stress-related ocular manifestations is still poorly explored, while current diabetic retinopathy therapy includes invasive methods, like surgery. Ocular antioxidant potential therapy represents a non-invasive, safe and less painful methodology, which slows the natural progress of the disease and improves the effectiveness of treatment without significant systemic toxicity. This review underlines the innovative medicines exploited for ocular conditions, a further insight on ocular delivery, benefiting from the advantages of the eye for drug delivery and, additionally, offering new potential applications of antioxidants for the prevention, treatment and control of diabetic retinopathy.
\end{abstract}

Keywords: Diabetes, Diabetic retinopathy; oxidative stress; Antioxidant activity

Abbreviations: CEL-N $\mathrm{N}^{€}$ : (carboxyethyl)lysine; CMA-N ${ }^{\epsilon}$ : (carboxymethyl)arginine; $\mathrm{CML}^{-} \mathrm{N}^{\epsilon}$-(carboxymethyl)lysine; GOLD: Glyoxal lysine dimer; $\mathrm{H}_{2} \mathrm{O}_{2}$ : Hydrogen peroxide; HNE: Hydroxynonenal; HOCL: Hypochlorous acid; MDA: Malondialdehyde; MDA-LDL: Malondialdehyde-modified Low Density Lipoprotein; MDA-Lys: Malondialdehyde lysine epitope; MnSOD: Maganese superoxide dismutase; MOLD: Methylglyoxal lysine dimer; NADPH: Reduced nicotinamide adenine dinucleotide phosphate

\section{Introduction}

Diabetes mellitus (DM) long-term complications are progressive and almost resulting by chronic exposure to high blood levels of glucose resulting from defects in insulin metabolism and dysfunction in carbohydrate, lipid and protein metabolism [1]. The diabetes complications are equally associated with the both types of DM. However, the severity of the disease is related to the longterm exposure to uncontrolled glycemia [2]. The incidence of DM, especially in industrialized countries has dramatically increased over the past two decades and it is expected to increase [3] in future. This disease has become one of the most challenging health problems of the 21 st century. It affects more than 230 million people worldwide, and this number is expected to reach 350 million by 2025 [1]. Globally the affected people are unaware of the disease and only half receive adequate treatment. It is therefore not surprising that diabetic retinopathy (DR) is the leading cause of blindness in people aged 2574 years worldwide [4-5].

The vascular commitment is the most serious and common condition in DM. Mediators of vascular damage of DM include poor glycemic control, lipoprotein abnormalities, hypertension, oxidative stress (OS), inflammation and advanced glycation end-products (AGEs), which are modified proteins formed by nonenzymatic glycation [6-7]. Pathological vascular dysfunction related with DM include DR like all diabetes conditions, is a progressive disease caused by chronic exposure to hyperglycemia, and recognized as a characteristic vascular disease [7], diabetic nephropathy (kidney) and diabetic neuropathy (peripheral nervous system) [5]. There is also evidence that hyperglycemia may induce diabetic angiopathy through the generation of OS [8], per se, or through the accumulation of AGEs, leading to nitrous oxide systems (NOS) [9].

Retinopathy is characterized by increased vascular permeability, by vascular closure mediated by the formation of new blood vessels - neovascularization, on the retina and posterior surface of the vitreous [10]. Generally, neovascularization results from occlusion of fragile capillaries and frequently originate pre-retinal and vitreous hemorrhage in case of vitreous detachment [11].

Without an effective medical treatment, cells and tissues of the retina become malnourished and progressively degenerate, which leads to damage in cells responsible for vision, leading to the inevitable loss of vision [5]. Approximately $25 \%$ of patients with type1 DM have been shown to be affected with retinopathy, with the incidence increasing to $60 \%$ after 5 years and $80 \%$ after 10 to 15 years of affliction [11]. The type-2 DM accounts for a higher proportion of patients with visual impairment [11]. Moreover, in general there are more adult onset cases than juvenile ones.

${ }^{*}$ Corresponding author: Sara Baptista da Silva, Department of Pharmaceutica Technology, Faculty of Pharmacy, University of Porto, Rua Aníbal Cunha 164 4050-047 Porto, Portugal, Tel: +351 222078 949; Fax: +351 222003 977; E-mail sara.baptistadasilva@gmail.com

Received November 09, 2010; Accepted December 07, 2010; Published December 09, 2010

Citation: da Silva SB, Costa JP, Pintado ME, Ferreira DC, Sarmento B (2010) Antioxidants in the Prevention and Treatment of Diabetic Retinopathy - A Review. J Diabet Metabol 1:111. doi:10.4172/2155-6156.1000111

Copyright: (C) 2010 da Silva SB, et al. This is an open-access article distributed under the terms of the Creative Commons Attribution License, which permits unrestricted use, distribution, and reproduction in any medium, provided the original author and source are credited. 
Although the pathogenesis of DR has not been clearly elucidated, multiple hypotheses have been proposed for this condition $[5,10]$.

However, more recently, much attention has been focused on the role of OS, and has been suggested that this may constitute the cause of different pathogenesis in diabetic complications, such as DR [3].

The retina is highly susceptible to OS because of intense exposure to light and oxygen and its high polyunsaturated fatty acid (PUFA) content that is prone to lipid peroxidation [12]. These oxidation products are toxic to the microvascular walls and therefore, may have a causal role in diabetic microvascular damage and also in the bloodocular barrier alteration [3]. Since OS is increased in the diabetic retina, the levels of oxidatively modified DNA and nitrosylated proteins are elevated, and antioxidant defense enzymes are impaired [13]. It has been reported that the level of antioxidant enzymes along with potential antioxidant vitamins are decreased in diabetic experimental animals and humans [10].Due to this intimate relationship between OS and dysmetabolisms implicated in the pathogenesis of DR, the use of appropriate antioxidants may have potential on the metabolic and functional abnormalities in DR. Antioxidants may act at different levels, including the inhibition of the formation of reactive oxygen species (ROS), scavenging free radicals, or increasing the antioxidants defense enzyme capabilities [1]. This suggests that potential management of diabetes could benefit from use of dietary or local biofactors of medicinal and aromatic plants. There is, therefore, research specificities to focus on the molecular mechanisms of action of the biofactors such as flavonoids, proanthocyanidins and alkaloids and/or extracts derived from plants [10].

Numerous drugs have been developed based on the current understanding of the complicated and intricate biochemical and pathophysiological aspects of the DR. However current therapy for DR includes laser photocoagulation, surgery, and metabolic control $[5,10]$. Having this in mind this paper suggests the high potential of antioxidants and the major importance of natural medicines to avoid the DR progression and consequent blindness.

\section{Ocular structure and permeability}

It is of extremely importance when considering eye diseases a deep knowledge of ocular structure so that it can be perceived what could be compromised in the eye ball, as well as the whole natural and complex process of ocular drug absorbing. This review chapter will start for a further insight in ocular anatomy and ocular permeability, so that the physiopathology and antioxidant activity could be easily comprehended in DR condition.

It is well known that the eyeball consists of two anatomical regions: the anterior segment, in which the cornea and conjunctiva are the main prominent structures, and the posterior segment, in which the retina plays the most important function on transduction and adaptation to different levels of light [14-15].

Anterior segment consists of front one-third of eye that mainly includes pupil, cornea, iris, ciliary body, aqueous humor, and lens while the posterior segment consists of the back two-thirds of the eye that includes vitreous humor, retina, choroid, macula, and optic nerve (Figure 1).

In the anterior segment of the eye, covering both corneal and conjunctival surfaces and forming part of the tear film, is a mucus layer, which is secreted by the goblet cells of the conjunctiva. The lachrymal film plays a multifunctional role, since it hydrates, cleanses, lubricates and serves as a defense against the pathogens. It is also involved in additional obstacle to any drug penetration, because the lachrymal film is a dynamic fluid that undergoes a constant renewal and therefore limits the time of residence of the drugs on the surface of the eye [15].

The cornea is a transparent and avascular hydrophobic barrier, continuous with the bulbar conjunctiva, organized into five layers: epithelium (a major lipophilic barrier), Bowman's membrane, stroma (a major hydrophilic barrier), Descemet's membrane, and endothelium (a minor lipophilic barrier). It is the primary route of drug entry into the eye following topical administration, but applied drugs also reach intraocular tissues by conjunctiva-scleral pathway [16]. Because of its nature, lipophilic drugs can easily cross the corneal epithelium by a transcellular pathway either by facilitated transport or by diffusion through the lipid bilayer $[15,17]$.

This tissue has a smaller surface area compared to the conjunctiva which, moreover, is a leakier epithelium than the cornea [18]. The conjunctiva is a mucus membrane that lines the inner surfaces of eyelids and folds back to cover the front surface of the eyeball, except for the central portion of the outer eye [19]. The conjunctiva covers most of the ocular surface area, only a small fraction (called the bulbar region) covers the anterior sclera, which has an air interface exposed to the environment during "open-eye" intervals. It continues through the fornicial and palpebral regions as highly vascularized, thin/semitransparent, elastic, and heterogeneous tissue [16]. Traditionally the role of the conjunctiva has been considered to be mainly protective and functioning as a passive physical barrier [15].

The conjunctiva can be divided into three layers, the outer epithelium, substantia propias, and submucosa and it has a higher permeability for large hydrophilic compounds that are transported via paracellular pathways. However, since lipophilic drugs mainly use a transcellular transport, the corneal route currently dominates [16, 18].

For eye protection the cornea and conjuctiva.epitheliums are considered similarly with respect to the organization of epithelial cells, including tight junctions in the apical pole and which makes them a relatively impermeable barriers [19]. Tight junctions provide a continuous seal around the epithelial cells, thereby preventing the entry of polar drug molecules into the subsequent tissues [19]. Other than cellular tight-junctional complexes in epithelium, the expression

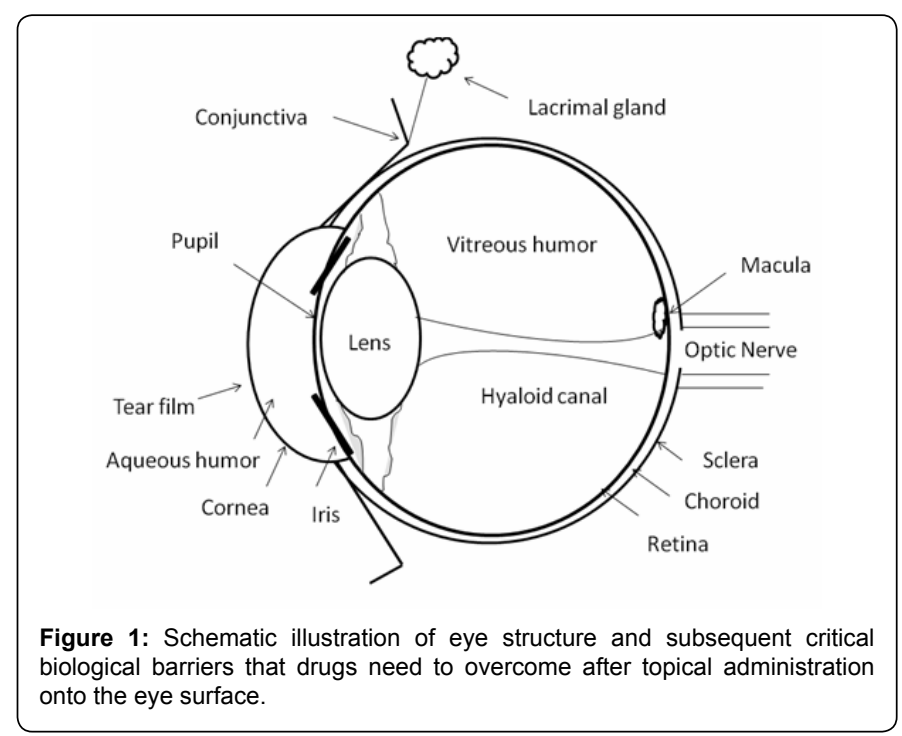




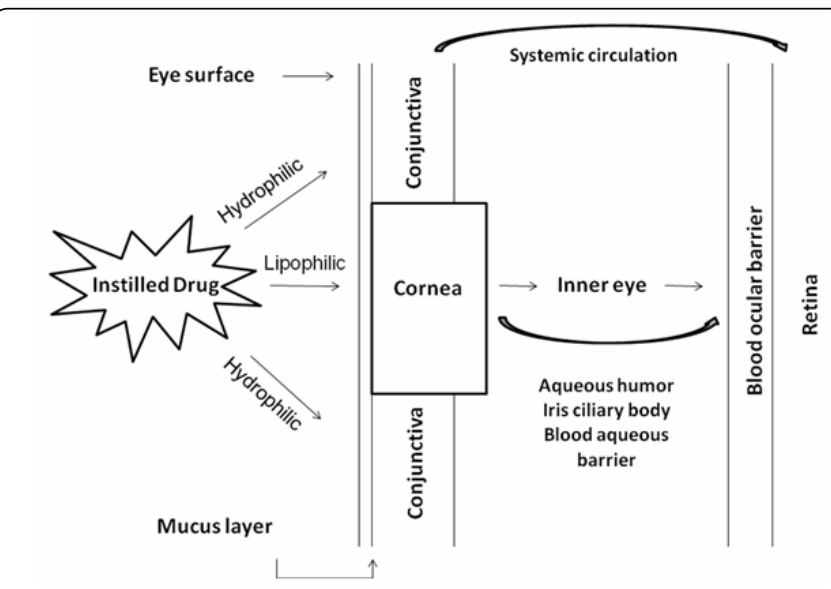

Figure 2: Schematic view of the ocular permeability, physiological barriers, and transport pathways of a drug applied topically onto the eye (modified from ref. [26]).

of efflux transporters is another important factor that contributes to the barrier properties, such as P-glycoprotein efflux pump (P-gp), and multi-drug-resistance protein (MRP) [15,17].

The retina is a thin, transparent, highly organized structure of neurons, glial cells, and blood vessels. Of all structures within the eye, the neurosensory retina has been the most widely studied. In short, the retina can be divided into neural retina and retinal pigment epithelium (RPE) [19]. The first is involved in signal transduction, leading to vision. Light enters through the retinal ganglion cell layer and penetrates all the layers. The transduced signal is taken by several retinal neurons to the optic nerve. The latter then drives the signal to the brain, where it is registered and formed an image. RPE, on the other hand, is a single cell layer that separates the outer surface of the choroid and neural retina and appears as a uniform, continuous layer that extends through the retina and a have an essential role in the viability of the neural retina [19].

Limited permeation into the retina primarily occurs due to the blood-aqueous barrier (BAB) and blood-retinal-barrier (BRB). These two barriers together comprise the blood-ocular barrier [19].

In addition to the physical barriers, ocular tissues contain metabolic enzymes, such as esterases, aldehyde and keton reductases [20-21], which may degrade the drugs and reduce their efficacy. As a result of these anatomical and physiological constraints [22] after topical application, a major fraction of the administered drug is lost by different mechanisms, resulting in very low ocular bioavailability.

Only about $1-5 \%$ of a topically applied drug dose often reaches the anterior segment of the eye. Therefore, subsequent diffusion of absorbed drugs toward the posterior segment of the eye will often be relatively low. Moreover eye drops in solution are eliminated from the pre-corneal area within 90 seconds and absorbed systemically through the highly vascular conjunctival stroma and nasolachrymal ducts [16].

This means that drugs topically administered have a low probability of reaching the posterior segment in significant amounts, as they have to pass through several metabolic and physical barriers to reach the retina, namely the corneal and conjunctival epithelium, then aqueous humor, and lens [19] (Figure 2). As shown in Figure 2, one of the main problems encountered with the topical administration of liquid forms is the rapid and extensive loss of drugs to the limited capacity of drug retention in the ocular surface and also as a result of the blinking process, which normally is stimulated after instillation. Obviously, the extent to which topically applied drugs remain on the surface of the eye or penetrate inside the eye is dependent not only on the physiological characteristics of the corneal barrier, but also on the physicochemical properties of the drug and the specific behavior of the vehicle.

Contrary to lipophilic drugs, hydrophilic drugs can be easily formulated as aqueous eye-drop solutions. Moreover, hydrophilic drugs have great difficulties in passing from the tear film to the corneal/ conjunctival epithelia. The paracellular entry through both corneal and non-corneal epithelia becomes the most important penetration route for hydrophilic drugs [23]. As regards to the absorption by the non-corneal route, studies have conclusively proved that the conjunctiva is a leakier epithelium that plays an important role in the absorption of large hydrophilic molecules $[16,18]$.

Altogether, for the successful treatment of pathologies that affect the posterior segment of the eye, improving the corneal and/or conjunctival tissues permeability becomes one of the main challenges in ocular drug delivery. Alternative routes other than topical, i.e. the systemic route or intrachameral injections, have also been considered for increasing the bioavailability of drugs in the internal structures of the eye. In the case of systemic administration, a major drawback is that only $1-2 \%$ of the administered drug reaches the vitreous cavity [15], especially blocked by the BRB, which is selectively permeable to more lipophilic molecules and mainly governs the entry of drug molecules into the posterior segment of the eye [24]. This results in frequent administration of high amounts of drug that may lead to important systemic side effects [25]. Some alternatives involve either injections of a drug, a drug delivery carrier or a drug delivery device into the vitreal cavity of the eye, or a periocular delivery following a transcleral route to the back of the eye that allows it to penetrate the RPE [17].

Local and new therapeutic improvements involving routes of administration easier and with a better performance upon biological barriers are essential for ocular delivery and effectiveness.

\section{Diabetic retinopathy}

The major risk factors for DR are known to be predominantly the hyperglycemia and the increased duration of diabetes. Other risk factors include hypertension, hyperlipidemia, pregnancy, and microalbuminuria [27-28]. All of these risk factors contribute and exacerbate retinal metabolic changes and microvascular injury that result in DR condition. Intensive glycemic control, like in other diabetes conditions, substantially reduces the incidence and progression of DR in type I and II diabetes [29], as well as blood pressure control [30]. Evidences of a causal relationship between insulin resistance and hypertension is increasing [31]. It is also increasingly clear that antihypertensive medications have disparate effects on insulin sensitivity in patients with essential hypertension, which makes the administration of specific antihypertensive agents relevant in these patients [32-34].

Abnormalities in retinal metabolism, including elevated polyol pathway activity [35], increased nonenzymatic glycation [36], accumulation of AGEs [5], uncontrolled OS [37], protein kinase C activity (PKC) [13] and the expression of vascular endothelial growth factor (VEGF) [13], result from glucose dysmetabolism and evidently also contribute to the development of retinopathy. However the exact mechanism is still elusive. 
The initial disease is characterized by increased vascular permeability due to a breakdown in the blood-retinal barrier (BRB), which causes macular edema, with a progressive vascular occlusion and retinal neovascularization [38].

Medical diagnosis, support, advices and treatment are fundamental, to avoid the malnourished and degenerative retinal cells/tissues, which leads to damage in the cells responsible for vision [5]. The clinical profile of this condition is chronologically subdivided in two stages, nonproliferative (NPDR) and the proliferative diabetic retinopathy (PDR). NPDR is the initial stage of the disease progression. Loss of retinal capillary pericytes and endothelial cells has been demonstrated early in diabetes [39] and underlies the clinical signs of NPDR, which include intra-retinal dot-blot hemorrhages, microaneurysms, and venous beading [13]. At this stage, blood and fluid leak from the blood vessel into the retinal tissue, resulting in retinal swelling and the formation of lipoprotein exudates deposits [5]. When neuronal cells in the retina begin to be compromised the process of neurodegeneration begins and culminates in more advanced stages of diabetic retinopathy.

The retinal edema is asymptomatic, if it occurs outside of the macula, but will impair vision when the macula is affected $[13,29]$. With progression of the disease to moderate or severe NPDR, blood vessels may be blocked in the retina, which, in turn, causes ischemia, hypoxia and deprival of nutrient nourishment in the affected area.

This blockade subsequently leads to pathologic growth of new blood vessels, which often causes catastrophic loss of vision and featuring the main serious stage of the disease - PDR [3,40,41]. The occlusion of capillaries in retinopathy induces angiogenesis in the afflicted retina, leading to the release of VEGF and insulinlike growth factor (IGF), which induce growth of new vessels on the optic disk, iris, retinal surface and into the vitreous, known as retinal neovascularization $[13,29]$. New blood vessels are fragile and may hemorrhage into the vitreous or form fibrous bands, causing tractional retinal detachment $[5,29]$. Neovascularization of the iris may occlude aqueous outflow, resulting in neovascular glaucoma [29]. New vessels are sometimes accompanied by a fibrovascular ridge extending into the vitreous cavity or along the surface of retina $[40,41]$. As microvascular damage weakens the BRB, plasma leaks from vessels into the retina; when this fluid is reabsorbed, lipid and lipoprotein elements are retained in the retina and are visible as yellow exudates [29]. Thus, retinal detachment may occur and lead to vision loss and blindness [40,41]. These features are detectable by ophthalmoscopy, because the pigment (hemoglobin) in blood or the lipid exudates stand out in contrast to the otherwise transparent retina. These changes have led to the general assumption that DR is solely a microvascular abnormality [42].

Most patients with DR are asymptomatic until very late stages of the disease [13]. The clinical signs of PDR are similar to those of NPDR in venous dilation, venous beading, and abnormal microvasculatures, and patients may complain about glare, blurred and vision loss [5].
The progression and evolution of the disease depends largely on glycemic control, medical surveillance, exposure to the disease and patient natural genetic predisposition.

If used appropriately, a number of tests ancillary to the clinical examination may enhance patient care. The most common tests include direct and indirect ophthalmoscopy, stereoscopic color film fundus photography, optical coherence tomography, fluorescein angiography and mydriatic or nonmydriatic digital color or monochromatic photography, ultrasonography [43].

Medical intervention and treatments for DR at present are focused on metabolic control of blood glucose and blood pressure and surgical procedures [29] which includes laser photocoagulation, surgery and the commonly intravitreal injection (IVT) [5].

\section{Oxidative stress clinical impairment}

In diabetes, the retina exhibit increased os $[37,44]$ since the eye is constantly subjected to light irradiation, atmospheric oxygen, environmental chemicals, and physical abrasion. The retina has also a natural high content of PUFA and possess the highest oxygen uptake and glucose oxidation relative to any other tissue, which makes it more susceptible to OS than other organs or structures [1]. All these factors, if not controlled induces can ultimately contribute to ocular surface damage and disease. Natural protective components like water-soluble antioxidants (e.g., vitamin C, L-cystine, reduced glutathione - GSH, uric acid, pyruvate, and tyrosine), lipid-soluble antioxidants (e.g., tocopherols and retinols), and highly specialized enzymes (e.g., superoxide dismutase - SOD, catalase, and Gluthathione peroxidase - GPx) have all been identified in human tear fluid collected at normal and stimulated secretion rates. These components are thought to serve as a frontline defense for the ocular surface tear film and underlying tissues. However, mechanisms or glandular sources of these antioxidants have not been identified [16].

So it can be clearly assumed that OS and ROS maybe a causal link between elevated glucose and the important metabolic abnormalities in the development of DR [45] (Table 1).

Free radicals are defined as an atoms or molecules that contain one or more unpaired electrons, making them unstable and highly reactive [46]. ROS are produced continuously in all cells to support normal cellular functions. Under normal physiological conditions, approximately $0.1 \%-5 \%$ of oxygen that enters the electron transport chain is reduced to superoxide, ROS and the rest is used in metabolic processes [1]. ROS can also be generated from other sources including cytochrome $\mathrm{P} 450$, the $\mathrm{NAD}(\mathrm{P}) \mathrm{H}$ oxidase(s) and Nitric Oxid (NO) synthases [47].

Excess production of ROS originated from endogenous or exogenous sources, and/or inefficient removal by scavenging system of ROS, can result in excessive levels of either molecular oxygen or ROS, thus resulting in increased OS, which often leads to damage of cellular macromolecules and destruction of small antioxidant

\begin{tabular}{|c|l|}
\hline \multicolumn{2}{|c|}{ Sources of oxidative stress } \\
\hline Sources of vascular oxidative stress & $\begin{array}{l}\text { Acute and chronic hyperglycaemia; Lipoprotein abnormalities; Hypertension; OS; Inflammation and AGEs accumulation; } \\
\text { Glycation; Glycoxidation; Lipoxidation; Extracellular metal ions; Superoxide and HOCl; NADPH/NADH oxidase; NO } \\
\text { synthase; Cyclooxygenase; Lipoxygenase; Myeloperoxidase; P450 monooxygenases; Enzymes of mitochondrial oxidative } \\
\text { phosphorylation; }\end{array}$ \\
\hline Increased generation of ROS & $\begin{array}{l}\text { Autoxidation of carbohydrates, fatty acids, triglycerides, phospholipids and cholesteryl esters; Acute and chronic } \\
\text { hyperglycemia; Glycation, advanced glycation and glycoxidation; }\end{array}$ \\
\hline Alterations in enzymatic pathways & $\begin{array}{l}\text { Increased polyol pathway activity; Decreased glyoxalase pathway activity; Alteration in mitochondrial oxidative metabolism; } \\
\text { Altered prostaglandin and leukotriene metabolism; }\end{array}$ \\
\hline Other mechanisms & Ischemia-reperfusion injury, hypoxia and pseudohypoxia; \\
\hline
\end{tabular}

Table 1: Possible conditions that may be sources of oxidative stress in diabetes mellitus (modified from ref. [46]). 


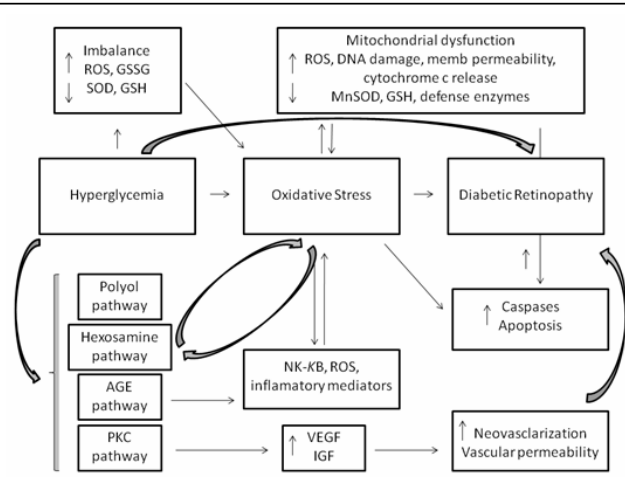

Figure 3: Oxidative stress-mediated dysmetabolisms in diabetic retinopathy (modified from ref. [1]).

Antioxidants

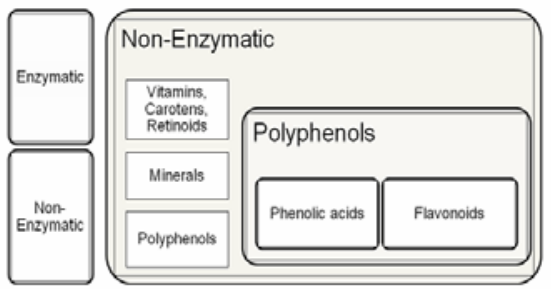

Figure 4: Antioxidants structure classification.

molecules $[1,46]$. These events are central to the pathogenesis of diabetes and its complications $[5,48]$. Major ROS include superoxide, peroxynitrite, NO and a combination of superoxide and NO [5]. These ROS oxidize proteins, lipids (lipoxidation), and carbohydrates (glycoxidation). The resulting oxidized molecules can cause further oxidative damage to cells leading to structural and functional changes of cellular organelles, especially when oxidized molecules accumulate intracellularly [46].

In general, in non-diabetic people these reactive species are effectively eliminated of several intracellular and extracellular antioxidant systems. However, in diabetes the increased intracellular concentrations of ROS appear to overwhelm the ability of many cells to neutralize radicals [48]. So the OS seems to be caused by an imbalance between the high increased production of ROS and the sharp reduction in antioxidant defenses and altered cellular redox status [46]. Antioxidant defenses are further depleted by other mechanisms, but it is initiated by high glucose levels. This includes increases in the polyol pathway whereby aldose reductase, reduces glucose to sorbitol at the expense of NADPH, usually used for regeneration of GSH from glutathione disulfide (GSSG) [49]. Damage from ROS and depleted cellular antioxidant defenses is manifested in several tissues, including the kidney, eye, and nervous system, for which the end results are often end-stage renal disease, blindness, and limb amputation, respectively [48].

In retinopathy, OS has been widely involved in decreased retinal blood flow [50], increased vascular permeability, disruption of BRB [51] and the appearance of acellular capillaries from the apoptotic loss of retinal capillary cells [52]. OS has also been linked to microvascular abnormalities in DR, degenerative process of retinal neovascularization, and the suppression of antioxidants systems [53]. Activation of the proapoptotic enzyme, caspase-3, and nuclear transcriptional factor-kappaB (NF- $\kappa \mathrm{B})$ occurred in the retinas of diabetic rats and in pericyte exposed to high glucose levels [53]. Diminished endogenous scavengers of oxidants and decreased activities of glutathione reductase (GR), GPx, SOD and catalase occur in the diabetic retina when compared to controls [54]. The overexpression of the cell death protease gene in retinal pericytes in diabetes is reported to correlate with the altered gene profile of the scavenging enzymes, suggesting an important role of OS in pericyte dropout seen in DR [13]. Further, increased OS has been shown to play a critical role in AGEs products-induced apoptosis of retinal capillary cells [55]. ROS can indirectly induce apoptosis by changing cellular redox potentials, depleting GSH and reducing ATP levels [56]. The release of ROS also increases mitochondrial pore permeability that in turn triggers the release of cytochrome $\mathrm{c}$ and other proapoptotic factors from retinal mitochondria, initiating apoptosis via activation of caspases [57-58] and increased cytochrome c is observed in the retina and its capillary cells in diabetes $[13,59]$. ROS-induced mitochondrial dysfunction pertaining to the release of cytochrome c can result in activation of caspase-9, which initiates a cascade of events that activates caspase-3 responsible for fragmenting DNA [58].

OS creates a vicious cycle of damage to macromolecules by amplifying the production of more ROS and also activates other metabolic pathways that are detrimental to the development of DR (Figure 3). These include the polyol pathway [60], AGE pathway [61], PKC pathway [62], hexosamine biosynthesis pathway [63], alteration in the expressions of VEGF [64], IGF-1 [65] and elevation in mitochondrial overproduction of superoxide and mitochondrial dysfunctions [13]. It is worthy to mention the various factors related to DR and underline the prominent importance of OS in the development and progression of DR. Several diabetes-induced abnormalities in the retina that are postulated in the development of retinopathy are influenced by OS and are considered to be interrelated [1].

Treatment with antioxidants is associated with partial restoration of diminished pericytes in retinal vessels in diabetic rats $[5,66]$. Moreover, the pericytes isolated from the retinas of diabetic donors highly expressed death proteases in the apoptotic pathway. These results clearly suggest that the OS is linked to apoptosis of retinal capillary cells and involved in microvascular cell loss during the course of DR [67].

Several biomarkers of DNA oxidation, lipid peroxidation, amino acids oxidation, glycoxidation and lipoxidation reactions have been identified and can be measured in short-lived intracellular proteins, plasma proteins, long-lived extracellular proteins and in urine by chemical methods (Table 2). These markers, measured by sensitive high-performance liquid chromatography or gas chromatographymass spectrometry, enzyme linked immuno sorbent assay (ELISA) and immunochemical techniques, are becoming an increasingly important part of the methodology for detection and measurement of oxidation products in tissues [46].

The etiology of OS and the subsequent issues of the oxidative tissue damaging have been widely exploited. However, the exact mechanism by which OS could contribute to the development of diabetic complications still remains to be clarified. Table 1 summarizes some possible issues associated with OS, diabetes deficient metabolism, as well as the possible sources of vascular OS in vascular smooth muscle cells and endothelial cells.

\section{Antioxidants as potential therapeutic agents}

Wellestablished antioxidants derived from the diet are vitamins C, E, A, and carotenoids, which have been studied intensively 


\begin{tabular}{|l|l|}
\hline \multicolumn{2}{|c|}{ Biomarkers of oxidative stress } \\
\hline ROS and RNS & $\begin{array}{l}\text { SOD radical, H2O2, NO, HOCL, peroxides, peroxyl radical, peroxynitrite, metal-oxo complexe, semiquinone radical, heme } \\
\text { proteins, singlet oxygen; }\end{array}$ \\
\hline Products of lipid peroxidation & MDA, 4-HNE, hydroperoxides, conjugated dienes, F2-isoprostanes dicarboxylic acids; \\
\hline Products of DNA oxidation & Modified bases, 8-oxo-2' deoxyguanosine, strand breaks; \\
\hline Primary products of protein oxidation & $\begin{array}{l}\text { o-tyrosine, o,o.-dityrosine, 3 chlorotyrosine,3-nitrotyrosine, dihydroxyphenylalanine, protein disulfides, methionine sulfoxide, } \\
\text { hydroperoxides of isoleucine, leucine, valine, protein carbonyls adipic semialdehyde, 2-oxohistidine; }\end{array}$ \\
\hline $\begin{array}{l}\text { Secondary products of protein } \\
\text { oxidation }\end{array}$ & $\begin{array}{l}\text { AGEs, ALEs, EAGLEs, Pentosidine, MDA-Lys, MDA-LDL, CML, CMA, CEL, Crosslines HNE (Lys, His, Cys) Argpyrimidine, } \\
\text { Vesperlysines, Pyrroles, GOLD, MOLD; }\end{array}$ \\
\hline $\begin{array}{l}\text { Antioxidant defense systems and } \\
\text { total antioxidant status }\end{array}$ & Levels of enzymes and antioxidants; \\
\hline
\end{tabular}

Table 2: Biomarkers of oxidative stress in diabetes mellitus (adapted from ref. [46]).

\begin{tabular}{|c|c|c|}
\hline Antioxidant & Mechanism of Action & References \\
\hline Aminoguanidine & $\begin{array}{l}\text { Inhibits the accelerated death of retinal capillary cells and development of retinopathy, inhibits lipid } \\
\text { peroxidation and AGEs formation; }\end{array}$ & {$[85-87]$} \\
\hline $\begin{array}{l}\text { Ascorbic acid, acetate, } \alpha \text { - tocopherol, trolox } \\
\text { cysteine, NAC, } \beta \text {-carotene and selenium }\end{array}$ & Reduce PKC in the retina, and lipid peroxides, prevents the drecrease of SOD, GR and catalase; & {$[12,77-78]$} \\
\hline Benfotiamine & $\begin{array}{l}\text { Inhibits MnSOD, increases the inhibiton of acellular capillaries in the retina blocking the major pathways } \\
\text { involved in hyperglycemia induced retinal dysmetabolism; }\end{array}$ & {$[88]$} \\
\hline Caffeic acid & $\begin{array}{l}\text { Anti-angiogenic activity in retinal endothelial cells and retinal neovascularization, suppression of the } \\
\text { ROSinduced and VEGF expression; }\end{array}$ & {$[81,97]$} \\
\hline Calcium dobsilate & Decreases retinal permeability, stabilized BRB and reduced overexpression of VEGF; & {$[11,79-80]$} \\
\hline Curcumin & Ocular anti-inflammatory multipotent activities; & {$[44,91]$} \\
\hline Lipoic acid & $\begin{array}{l}\text { Attenuates the apoptosis of retinal capillary cells and acellular capillaries, decreases the levels of } \\
\text { nitrotyrosine, VEGF and oxidatively modified proteins, activation of NF-kB; }\end{array}$ & {$[73,83]$} \\
\hline Nicanartine & Inhibits pericyte loss; & [8] \\
\hline Pycnogenol & Free radical scavenger, antiinflammatory properties; & [92] \\
\hline Pyridoxamine & Inhibits the formation of diabetes-induced retinal acellular strands; & {$[88]$} \\
\hline Rosmarinic acid & $\begin{array}{l}\text { Anti-angiogenic activity to retinal neovascularization, inhibits the proliferation of retinal endothelial cells, } \\
\text { and the angiogenesis of tube formation; }\end{array}$ & [84] \\
\hline Selenium & Down-regulate VEGF production in the retina of diabetics; & [90] \\
\hline Trolox & Partially prevents the loss of pericytes via reducing membrane lipid peroxidation; & [76] \\
\hline Vitamin $\mathrm{C}$ and $\mathrm{E}$ & Reduce neovascularization, prevent the inhibition of retinal GR, GPx and SOD activities; & {$[76-77,98]$} \\
\hline Vitamin E & Restores retinal blood flow, free radical scavenger, normalize diabetic retinal hemodynamics; & {$[46,48,50]$} \\
\hline Zinc & Prevents diabetes-induced GSH loss in the retina; & [89] \\
\hline
\end{tabular}

Table 3: Antioxidants described to have high therapy potential in diabetic retinopathy and their way of action.

[68]. Vegetables and fruits have in their natural composition other substances besides these antioxidant vitamins which guarantees health benefits associated with its consumption. Over the past decade evidence has been accumulated that plant polyphenols are an important class of defense antioxidants (Figure 4). These compounds are widespread virtually in all plant foods, often at high levels, and include phenols, phenolic acids and flavonoids [68].

Antioxidants may act at different levels, inhibiting the formation of ROS or scavenge free radicals, or increase the antioxidants defense enzyme capabilities $[1,4,69]$ (Table 3 ).

However, in the case of macrovascular/microvascular complications the antioxidant therapy is beneficial together with blood pressure control, management of dyslipidemia and optimal glucose control [46].

Generally, the antioxidant pharmacotherapy can be divided in the use of antioxidant enzyme and substrates, biogenic elements, combined drugs, synthetic antioxidants and drugs with antioxidant activity. There are also a large number of natural cellular defense mechanisms as the naturally existing antioxidant components, which neutralizes free radical damage. The enzymatic antioxidant systems, such as Copper $(\mathrm{Cu})$, Zinc $(\mathrm{Zn})$, manganese superoxide dismutase (MnSOD), GPx, GR and catalase may remove the ROS directly or sequentially, preventing their excessive accumulation and consequent adverse effects [30]. Non-enzymatic antioxidant systems consist of scavenging molecules that are endogenously produced such as GSH, ubichinol and uric acid or derivatives of the diet such as vitamins $\mathrm{C}$ and $\mathrm{E}$, carotenoids, lipoic acid, selenium, etc [70].

The consideration for both - the intake of antioxidants special monitoring through diet or through supplementation should include a careful analysis of data, including the role of antioxidants physiologically relevant as well as the concentrations to alleviate diabetic complications, with minimal side effects. Indeed, antioxidants such as $\mathrm{N}$-acetylcysteine (NAC) [71], vitamin C [72] and $\alpha$-lipoic acid [48] are effective in reducing diabetic complications, indicating that it may be beneficial either by ingestion of natural antioxidants or through dietary supplementation [73].

Administration of antioxidants to diabetic rats is able to prevent the development of retinopathy and also retinal metabolic abnormalities postulated to be involved in the development of retinopathy $[74,75]$. Following positive results of the prevention of diabetes-associated vascular dysfunction in a diabetic rat model, high doses of vitamin $\mathrm{E}$, the major antioxidant in lipid phase were studied in the clinic and found to restore retinal blood flow in diabetic type I patients to control levels $[48,50]$. The potential benefit of vitamin $\mathrm{E}$, has been shown in DR by its free radical scavenger activity outside the cell through nonenzymatic mechanisms[50]. Studies in humans suggested that antioxidant therapy with vitamin E might normalize diabetic retinal hemodynamics. [46]. Trolox is a water soluble analog of vitamin $\mathrm{E}$ with potent antioxidant properties. Trolox is shown to partially prevent the loss of pericytes in diabetic rats via reducing membrane lipid peroxidation [76].

Superoxide production in the retina was suppressed by a combination of vitamins $\mathrm{C}$ and $\mathrm{E}$ and partial reductions in neovascularization of the retina were observed in diabetic rats that received this vitamin combination [76]. Multiantioxidants supplementation of these vitamins in diabetic rats also prevents the inhibition of retinal GR, GPx and SOD activities [77]. The benefits related to the survival of retinal cells are more notorious when associated with the consumption of other antioxidants as ascorbic acid, acetate, $\alpha$-tocopherol, trolox cysteine, NAC, $\beta$-carotene and selenium $[12,77,78]$. The same components can also reduce the 
activity of PKC in the retina, reduces lipid peroxides and prevents the decrease of SOD, GR and catalase [77]. Thus, it is considered that increasing the application or consumption of a greater diversity of antioxidants is the best way to prevent retinopathy

Genetic engineering techniques demonstrated that overexpression of mitochondrial SOD in mice can decrease retinal OS and protect mitochondria dysfunction[67], which raises the possibility of a pharmacological approach attractive to inhibit the development of DR.

Another antioxidant, calcium dobsilate decreased retinal permeability, stabilized BRB and reduced overexpression of VEGF in diabetic rats [11]. Independent of diabetes control in the patients, calcium dobesilate, prevented BRB disruption [79]. These effects of calcium dobesilate appear to be related to its antioxidant activity. However, the precise mechanism of the drug-induced protection from DR is poorly understood, although it has been used to treat DR in many countries [80]. Caffeic acid has anti-angiogenic activity in retinal endothelial cells and retinal neovascularization, which may be related to suppression of the ROS-induced and VEGF expression [81] without retinal toxicity[82]. Suggesting that might be pharmacologically applied to vaso-proliferative retinopathy including DR, age macular degeneration (AMD), as well as retinopathy of prematurity (ROP). Lipoic acid attenuates the apoptosis of rat retinal capillary cells and decreases the levels of nitrotyrosine [83]. Lipoic acid supplementation completely prevents diabetes-induced increase in nitrotyrosine and activation of NF- $k B$ while decreasing the levels of VEGF and oxidatively modified proteins in the rat retina [83]. This antioxidant also inhibits diabetes-induced decreases in retinal mitochondrial and cytosolic ratios of NAD+ to NADH $[73,83]$. Rosmarinic acid has an anti-angiogenic activity to retinal neovascularization in a mouse model of retinopathy. Significantly inhibited the proliferation of retinal endothelial cells in a dose-dependent manner, and inhibited in vitro angiogenesis of tube formation. Moreover, rosmarinic acid showed no retinal toxicity. These data suggest rosmarinic acid could be a potent inhibitor of retinal neovascularization and may be applied in the treatment of other vasoproliferative retinopathies [84]. Aminoguanidine a compound with antioxidant potential has been found to inhibit the accelerated death of retinal capillary cells and development of retinopathy [85]. It also inhibits lipid peroxidation, AGEs formation in experimental models, and the development of nephropathy and neuropathy $[86,87]$. However, all clinical trials with aminoguanidine (Pimagidine) in Europe and then in the U.S. were discontinued due to its long-term toxicity.

Benfotiamine, a lipid soluble thiamine derivative that inhibits MnSOD, has been shown to increases the inhibition of acellular capillaries in the retina of diabetic rats via blocking the major pathways involved in hyperglycemia induced retinal dysmetabolism, including AGEs, PKC, and hexosamine pathways [88]. Pyridoxamine inhibits the formation of diabetes-induced retinal acellular strands in rats [88]. Nicanartine, an antioxidant with cholesterol lowering properties, can partially inhibit pericyte loss in diabetic rats. However, in the same animals it fails to provide any benefit in normalizing diabetes-induced increase in retinal acellular capillaries [8]. Zinc, a trace element with antioxidant properties, is shown to prevent diabetes-induced GSH loss in the retina [89]. Further, another trace element, selenium, is reported to down-regulate VEGF production in the retina in diabetes [90].

Owing to its antioxidative and anti-inflammatory multipotent activities, curcumin, a naturally occurring yellow pigment, could represent a preventive treatment option for inflammatory retinal diseases such as AMD, DR and light-induced retinal degeneration (LIRD)[44,91]. Pycnogenol, a compound with both free radical scavenging and anti-inflammatory properties, is also reported to have beneficial effects on the progression of retinopathy in diabetic patients [92].

Taurine and vitamin E supplementation along with selenium also reduced biochemical retinal alterations in diabetic rats [93]. Tempol is a membrane-permeable and metal-independent superoxide dismutase (SOD) mimetic and has been used for the removal of intracellular and extracellular free radicals and it seems that in obese Zucker rats, tempol reduced blood pressure, blood glucose, insulin, renal oxidative stress, and protein kinase $C$ activity [94].

Supplemental treatment based on extracts could be also very attractive since major compounds may work together, like in the green tea, which is highly rich in polyphenols with great antioxidant potency and inhibits lipid peroxidation, scavenges hydroxyl and superoxide radicals [76]. Green tea supplementation in diabetic rats reported an improvement of the SOD levels and GSH, reduction on the serum glucose levels, and reduce retinopathy signs as evident by reductions in acellular capillaries and pericyte ghosts [95]. Evidences also suggest that green tea significantly inhibited diabetic cataracts in streptozotocin-induced rat model of diabetes [96]. This provides encouraging rationale for its possible therapeutic use to inhibit retinopathy in diabetic patients.

As cited in this section, many agents may have antioxidant activity and protect the retina from the natural progression of DR (Table 3). However, more studies should be explored considering the topical and additional administration of antioxidants to patients with the first sights of the disease. Nevertheless, antioxidant therapy cannot be considered a unique action in the prevention and treatment of DR. It should be taken into account multiantioxidant administration or supplementation, as well as a regular diet, hyperglycemic control and medical surveillance.

\section{Emergent clinical trials}

The need to improve the ocular drug bioavailability, effectiveness and higher retention time becomes an emerging field in medicine, considering the exponential rise of diabetes worldwide and the consequent increase of DR condition. Although there are at least 60 agents in the clinic touted as anti-angiogenic, many more potential anti-angiogenic candidates are currently in preclinical development, with the distinct possibility of moving into ocular clinical studies $[97,98]$. On the other hand, and until now, no molecular therapies have received FDA approval for the treatment of diabetic macular edema and PDR $[4,99]$. Recent data from very important multicenter clinical trials have emphasized the importance of new and evolutional therapies. A clinical trial concluded that emerging treatments like protein kinase $C$ inhibitors and reduction of oxidative stress/ superoxide possibly used in combination with standard therapy, offer the hope of effective and safe treatment that may allow us to improve visual outcomes and prevent the damaging consequences of DR [100].

However and conciedering antioxidants therapy the results from clinical trials may seem ambiguous, since many drugs for DR control with antioxidant potential and actual effects in vivo, did not result when administrated in human volunteers $[5,97]$. The differences for such discrepancies are not clear, but it is possible that the initiation of antioxidants could be subsequent to the development of background 
retinopathy, in contrast to the animal studies where antioxidants have been administered soon after establishment of diabetes. Another reason for this discrepant results observed in clinical trial might be also due to antioxidants unspecific actions, or not so specific as it could be expected. The antioxidant concentrations in the retina could be also not sufficient to produce beneficial effects in humans [78,101].

However antioxidants appear to be promising in inhibiting the development of DR in animal models, but further clinical studies are needed to determine the appropriate regimen, and also whether these therapies could have long-term effects that may slow the progression of this sight-threatening complication of diabetes [1]. If the treatment does not completely inhibit the targeted metabolic abnormality, this could result in partial inhibition of other interrelated abnormalities. It would be prudent not have just one single drug that could effectively treat this complication of diabetes, but the use of a group of drugs with divergent mechanisms of action to combat this multifactorial complication, in which antioxidants could be an integral part of that healing [1].

\section{Conclusion}

The use of topical antioxidants to treat or delaying oxidative stress-related ocular manifestations is still unexplored, while current DR therapy includes invasive method like laser photocoagulation or surgery, which may also increase risk of endophthalmitis, cataract formation and retinal detachment. Besides the development of laser for DR, there have been no major advances in treatment for the disease, despite numerous clinical trials. Drugs applied directly to the eye represent a non-invasive and safe methodology, increasing the effectiveness of treatment and reducing toxicity associated with systemic administration.

An ideal anti-angiogenic agent should be developed for neovascularization control and regression. It may inhibit and stabilize the disease, to improve the vision loss and prevent retinal scarring and detachment with no toxicity as well as the formulation should be for long term drug delivery. Agents should also be classified into early and late acting, specific and non-specific, and reversible and irreversible. The understanding of where a drug falls into these classes may help in the comprehension of the potential and/or limitation of the drug when used in the clinic, as well as how to predict potential serious adverse events. Considering this, there are a number of challenges associated to the treatment of ocular diseases. In general, the major problem in ocular therapeutics is to maintain an effective drug concentration at the site of action for an appropriate period of time, in order to achieve the expected pharmacological response. Polymeric adhesive nanoparticles have been utilized to enhance the performance of common drugs, increasing drug time retention, slow drug delivery, with a specific target while reducing systemic side effects compared with commercial aqueous eye drops. The smart symbiosis of these adhesive particles with a high potent antioxidant could be a hope for future therapies, considering the important effect of OS in the pathogenesis of DR. This therapeutic improvement is expected to offer real benefits in the stability, bioavailability, drug delivery and therapy of the diabetic patients. Thus, successful alternatives for ocular therapies are needed and they should provide non-invasive and a cost effective treatment reaching every economic status.

\section{Acknowledgements}

Funding for author Sara B. Silva was via a PhD fellowship, administered by Fundação para a Ciência e a Tecnologia (SFRH/BD/61423/2009).

\section{References}

1. Kowluru RA, Chan PS (2007) Oxidative Stress and Diabetic Retinopathy. Exp Diabetes Res: 43603.

2. Shukla D, Rajendrana A, Singha J, Ramasamya K, Perumalsamya N, et al. (2003) Atypical manifestations of diabetic retinopathy. Curr Opin Ophthalmol 14: $371-377$

3. Yildirim Z, Ucgun NI, Kilic N, Gursel E, Sepici-Dinçel A (2007) Antioxidant Enzymes and Diabetic Retinopathy. Ann N Y Acad Sci 1100: 199-206.

4. Kalishwaralal K, BarathManiKanth S, Pandian SR, Deepak V, Gurunathan S (2010) Silver Nano - A Trove for Retinal Therapies. J Control Release 145: 76-90.

5. Chu J, Ali Y (2008) Diabetic Retinopathy: A Review. Drug Dev Res 69: 1-14.

6. Wiwanitkit $\mathrm{V}$ (2007) Oxidation flux change in diabetic retinopathy: does it exist or not? Diabetol Croat 36.

7. Soro-Paavonen A, Forbes JM (2006) Novel therapeutics for diabetic micro- and macrovascular complications. Curr Med Chem 13: 1777-1788.

8. Hammes HP, Bartmann A, Engel L, Wülfroth $P$ (1997) Antioxidant treatment of experimental diabetic retinopathy in rats with nicanartine. Diabetologia 40 : 629-634.

9. Santilli F, Cipollone F, Mezzetti A, Chiarelli F (2004) The role of nitric oxide in the development of diabetic angiopathy. Horm Metab Res 36: 319-335.

10. Aruoma OI, Neergheen VS, Bahorun T, Jen LS (2007) Free Radicals, Antioxidants and Diabetes: Embryopathy, Retinopathy, Neuropathy, Nephropathy and Cardiovascular Complications. Neuroembryol Aging 4: 117137.

11. Kumari S, Panda S, Mangaraj M, Mandal MK, Mahapatra PC (2008) Plasma and Antioxidant Vitamins in Diabetic Retinopathy. Indian J Clin Biochem 23: 158-162.

12. Terrasa AM, Guajardo MH, Marra CA, Zapata G (2009) Alpha-Tocophero protects against oxidative damage to lipids of the rod outer segments of the equine retina. Vet $\mathrm{J}$ 182: 463-468.

13. Kowluru RA (2005) Diabetic retinopathy: mitochondrial dysfunction and retina capillary cell death. Antioxid Redox Signal 7: 1581-1587.

14. Gaudana R, Jwala J, Boddu SHS, Mitra AK (2009) Recent perspectives in ocular drug delivery. Pharm Res 26: 1197-1216.

15. Fuente Mdl, Raviña M, Paolicelli P, Sanchez A, Seijo B, et al. (2010) Chitosanbased nanostructures: A delivery platform for ocular therapeutics. Adv Drug Deliv Rev 62: 100-117.

16. Gukasyan HJ, Kim KJ, Lee VHL (2008) The Conjunctival Barrier in Ocular Drug Delivery. Springer VII: $307-320$.

17. Ali M, Byrne ME (2008) Challenges and solutions in topical ocular drug-delivery systems. Expet Rev Clin Pharmacol 1: 145-161.

18. Hosoya K, Lee VH, Kim KJ (2005) Roles of the conjunctiva in ocular drug delivery: a review of conjunctival transport mechanisms and their regulation. Eur J Pharm Biopharm 60: 227-240.

19. Gunda S, Hariharan S, Mandava N, Mitra AK (2008) Barriers in Ocular Drug Delivery. Ocular Transporters in Ophthalmic Diseases and Drug Delivery VII: 399-413.

20. Duvvuri S, Majumdar S, Mitra AK (2004) Role of metabolism in ocular drug delivery. Curr Drug Metab 5: 507-515.

21. Barar J, Javadzadeh AR, Omidi Y (2008) Ocular novel drug delivery: impacts of membranes and barriers. Expert Opin Drug Deliv 5: 567-581.

22. Urtti A (2006) Challenges and obstacles of ocular pharmacokinetics and drug delivery. Adv Drug Deliv Rev 58: 1131-1135.

23. Ghate D, Edelhauser HF (2006) Ocular drug delivery. Expert Opin Drug Deliv 3: 275-287.

24. Mannermaa E, Vellonen KS, Urtti A (2006) Drug transport in corneal epithelium and blood-retina barrier: emerging role of transporters in ocular pharmacokinetics. Adv Drug Deliv Rev 58: 1136-1163.

25. Hughes PM, Olejnik O, Chang-Lin JE, Wilson CG (2005) Topical and systemic drug delivery to the posterior segments. Adv Drug Deliv Rev 57: 2010-2032. 
Citation: da Silva SB, Costa JP, Pintado ME, Ferreira DC, Sarmento B (2010) Antioxidants in the Prevention and Treatment of Diabetic Retinopathy - A Review. J Diabet Metabol 1:111. doi:10.4172/2155-6156.1000111

26. Alonso MJ, Sanchéz A (2003) The potential of chitosan in ocular grug delivery. J Pharm Pharmacol 55: 1451-1463.

27. Wong TY, Klein R, Islam A, Cotch MF, Folsom AR, et al. (2006) Diabetic retinopathy in a multi-ethnic cohort in the United States. Am J Ophthalmol 141 446-455

28. Mohamed QG, Wong TY (2007) Management of diabetic retinopathy: a systematic review. JAMA 298: 902-916.

29. Fante RJ, Durairaj VD, Oliver SCN (2010) Diabetic Retinopathy: An Update on Treatment. Am J Med 123: 213-216.

30. Matthews DR, Stratton IM, Aldington SJ, Holman RR, Kohner EM (2004) Risks of progression of retinopathy and vision loss related to tight blood pressure control in type 2 diabetes mellitus: UKPDS 69. Arch Ophthalmol 122: 1631 1640.

31. Sowers JR (2004) Insulin resistance and hypertension. Am J Physiol Heart Circ Physiol 286: H1597-H602.

32. Verdecchia P, Reboldi G, Angeli F, Borgioni C, Gattobigio R, et al. (2004) Adverse prognostic significance of new diabetes in treated hypertensive subjects. Hypertension 43: 963-969.

33. Stump CS, Hamilton MT, Sowers JR (2006) Effect of Antihypertensive Agents on the Development of Type 2 Diabetes Mellitus. Mayo Clin Proc 81: 796-806.

34. Lee BY, AL-Waili N, Stubbs D, Wendell K, Butler G, et al. (2010) Ultra-low microcurrent in the management of diabetes mellitus, hypertension and chronic wounds: Report of twelve cases and discussion of mechanism of action. Int J Med Sci 7: 29-35.

35. Naruse K, Nakamura J, Hamada Y, Nakayama M, Chaya S et al. (2000) Aldose reductase inhibition prevents glucose-induced apoptosis in cultured bovine retinal microvascular pericytes. Exp Eye Res 71: 309-315.

36. Stitt AW (2003) The role of advanced glycation in the pathogenesis of diabetic retinopathy. Exp Mol Pathol 75: 95-108.

37. Kowluru RA, Tang J, Kern TS (2001) Abnormalities of retinal metabolism in diabetes and experimental galactosemia. VII. Effect of long-term administration of antioxidants on the development of retinopathy. Diabetes 50: 1938-1942.

38. Barber AJ (2003) A new view of diabetic retinopathy: a neurodegenerative disease of the eye. Prog Neuropsychopharmacol Biol Psychiatry 27: 283- 290.

39. Frank RN (2004) Diabetic retinopathy. N Engl J Med 350: 48-58.

40. Ciulla TA, Amador AG, Zinman B (2003) Diabetic retinopathy and diabetic macular edema: pathophysiology, screening, and novel therapies. Diabetes Care 26: 2653-2664

41. Yam JC, Kwok AK (2007) Update on the treatment of diabetic retinopathy. Hong Kong Med J 13:46-60.

42. Gardner TW, Antonetti DA, Barber AJ, LaNoue KF, Levison SW (2002) Diabetic Retinopathy: More Than Meets the Eye. Surv Ophthalmol 47: 253-262.

43. American Academy of Ophthalmology Retina Panel. Preferred Practice Pattern ${ }^{\circledR}$ Guidelines. In: retinopathy D, editor. San Francisco: American Academy of Ophthalmology; 2008.

44. Kowluru RA, Kanwar M (2007) Effects of curcumin on retinal oxidative stress and inflammation in diabetes. Nutr Metab 4: 1-8.

45. Brownlee M (2001) Biochemistry and molecular cell biology of diabetic complications. Nature 414: 813-820.

46. Jakus $V(2000)$ The role of free radicals, oxidative stress and antioxidan systems in diabetic vascular disease. Bratisl Lek Listy 101: 541-551.

47. Droge W (2002) Free radicals in the physiological control of cell function Physiol Rev 82: 47-95.

48. Pazdro R, Burgess JR (2010) The Role of Vitamin E and Oxidative Stress in Diabetes Complications. Mech Ageing Dev 131: 276-286.

49. Lorenzi M (2007) The polyol pathway as a mechanism for diabetic retinopathy: attractive, elusive, and resilient. Exp Diabetes Res: 61038.

50. Bursell S, Clermont A, Aiello LP, Aiello LM, Schlossman D, et al. (1999) Highdose vitamin $\mathrm{E}$ supplementation normalizes retinal blood flow and creatinine clearance in patients with type 1 diabetes. Diabetes Care 22: 1245-1251.

51. Ellis EA, Guberski DL, Somogyi-Mann M, Grant MB (2000) Increased H 2 O 2 vascular endothelial growth factor and receptors in the retina of the BBZ/Wor diabetic rat. Free Radical Biol Med 28: 91-101.
52. Kern TS, Tang J, Mizutani M, Kowluru RA, Nagaraj RH et al. (2000) Response of capillary cell death to aminoguanidine predicts the development of retinopathy: comparison of diabetes and galactosemia. Invest Ophthalmol Vis Sci 41: 3972-3978.

53. Kowluru RA, Koppolu P (2002) Diabetes-induced activation of caspase-3 in retina: effect of antioxidant therapy. Free Radical Res 36: 993-999.

54. Leal EC, Santiago AR, Ambrosio AF (2005) Old and new drug targets in diabetic retinopathy: from biochemical changes to inflammation and neurodegeneration. Curr Drug Targets: CNS Neurol Disord 4: 421-434.

55. Denis U, Lecomte M, Paget C, Ruggiero D, Wiernsperger N, et al. (2002) Advanced glycation end-products induce apoptosis of bovine retinal pericytes in culture: involvement of diacylglycerol/ceramide production and oxidative stress induction. Free Radical Biol Med 33: 236-247.

56. Hancock JT, Desikan R, Neill SJ (2001) Does the redox status of cytochrome C act as a fail-safe mechanism in the regulation of programmed cell death? Free Radical Biol Med 31: 697-703.

57. Anuradha CD, Kanno S, Hirano S (2001) Oxidative damage to mitochondria is a preliminary step to caspase-3 activation in fluoride-induced apoptosis in HL60 cells. Free Radical Biol Med 31: 367-373.

58. Phaneuf S, Leeuwenburgh C (2002) Cytochrome c release from mitochondria in the aging heart: a possible mechanism for apoptosis with age. Am J Physio Regul Integr Comp Physiol 282: R423-R430.

59. Kowluru RA, Kowluru V, Xiong Y, Ho Y-S (2006) Overexpression of mitochondrial superoxide dismutase in mice protects the retina from diabetesinduced oxidative stress. Free Radical Biol Med 41: 1191-1196.

60. Engerman RL, Kern TS, Larson ME (1994) Nerve conduction and aldose reductase inhibition during 5 years of diabetes or galactosaemia in dogs. Diabetologia 37: 141-144.

61. Beisswenger PJ, Howell SK, Smith K, Szwergold BS (2003) Glyceraldehyde-3 phosphate dehydrogenase activity as an independent modifier of methylglyoxal levels in diabetes. Biochim Biophy Acta 1637: 98-106.

62. Koya D, King GL (1998) Protein kinase C activation and the development of diabetic complications. Diabetes 47: 859-866.

63. Du XL, Edelstein D, Rossetti L, Fantus IG, Goldberg H, et al. (2000) Hyperglycemia-induced mitochondrial superoxide overproduction activates the hexosamine pathway and induces plasminogen activator inhibitor-1 expression by increasing Sp1 glycosylation. Proc Natl Acad Sci USA 97: 12222-12226.

64. Caldwell RB, Bartoli M, Behzadian MA, El-Remessy AE, Al-Shabrawey M, et al. (2005) Vascular endothelial growth factor and diabetic retinopathy: role of oxidative stress. Curr Drug Targets 6: 511-524.

65. DeBosch BJ, Baur E, Deo BK, Hiraoka M, Kumagai AK (2001) Effects of insulin-like growth factor-1 on retinal endothelial cell glucose transport and proliferation. J Neurochem 77: 1157-1167.

66. Ansari NH, Zhang W, Fulep E, Mansour A ( 1998) Prevention of pericyte loss by trolox in diabetic rat retina. J Toxicol Environ Health 54: 467-475.

67. Kowluru RA, Atasi L, Ho YS (2006) Role of mitochondrial superoxide dismutase in the development of diabetic retinopathy. Invest Ophthalmol Vis Sci 47: 15941549 .

68. Pietta PG (2000) Flavonoids as Antioxidants. J Nat Prod 63: 1035-1042.

69. Frank RN (2002) Potential new medical therapies for diabetic retinopathy: protein kinase C inhibitors. Am J Ophthalmol 133: 693-698.

70. Matteucci E, Giampietro O (2000) Oxidative stress in families of type 1 diabetic patients. Diabetes Care 23: 1182-1186.

71. Steiner G (2000) Lipid intervention trials in diabetes. Diabetes Care 23 B49-B53.

72. Zherebitskaya E, Akude E, Smith DR, Fernyhough P (2009) Development of selective axonopathy in adult sensory neurons isolated from diabetic rats: role of glucose-induced oxidative stress. Diabetes 58: 1356-1364

73. Lin J, Bierhaus A, Bugert P, Dietrich N, Feng F, et al. (2006) Effect of R-(+)alpha-lipoic acid on experimental diabetic retinopathy. Diabetologia 49: 1089 1096

74. Fennell JP, Brosnan MJ, Frater AJ, Hamilton CA, Alexander MY, et al.(2002) Adenovirus-mediated overexpression of extracellular superoxide dismutase improves endothelial dysfunction in a rat model of hypertension. Gene The 9: 110-117. 
Citation: da Silva SB, Costa JP, Pintado ME, Ferreira DC, Sarmento B (2010) Antioxidants in the Prevention and Treatment of Diabetic Retinopathy - A Review. J Diabet Metabol 1:111. doi:10.4172/2155-6156.1000111

75. Zhu HL, Stewart AS, Taylor MD, Vijayasarathy C, Gardner TJ, et al. (2000) Blocking free radical production via adenoviral gene transfer decreases cardiac ischemia-reperfusion injury. Mol Ther 2: 470-475.

76. Mustata GT, Rosca M, Biemel KM, Reihl O, Smith MA, et al. (2005) Paradoxical effects of green tea (Camellia sinensis) and antioxidant vitamins in diabetic rats: improved retinopathy and renal mitochondrial defects but deterioration of collagen matrix glycoxidation and cross-linking. Diabetes 54: 517-526.

77. Kowluru RA, Tang J, Kern TS (2001) Abnormalities of retinal metabolism in diabetes and experimental galactosemia. VII. Effect of long-term administration of antioxidants on the development of retinopathy. Diabetes 50: 1938-1942.

78. Millen AE, Gruber M, Klein R, Klein BEK, Palta M, et al. (2003) Relations of serum ascorbic acid and $\alpha$-tocopherol to diabetic retinopathy in the Third National Health and Nutrition Examination Survey. Am J Epidemiol 158: 225233

79. Rota R, Chiavaroli C, Garay RP, Hannaert P (2004) Reduction of retinal albumin leakage by the antioxidant calcium dobesilate in streptozotocin-diabetic rats. Eur J Pharmacol 495: 217-224.

80. Ribeiro ML, Seres AI, Carneiro AM, Stur M, Zourdani A, et al. (2006) Effect of calcium dobesilate on progression of early diabetic retinopathy: a randomised double-blind study. Graefes Arch Clin Exp Ophthalmol 244: 1591-1600.

81. Paynter NP, Yeh HC, Voutilainen S, Schmidt MI, Heiss G, et al. (2006) Coffee and sweetened beverage consumption and the risk of type 2 diabetes mellitus: the atherosclerosis risk in communities study. Am J Epidemiol 164: 1075-1084.

82. Kim JH, Lee BJ, Yu YS, Kim KW (2010) Anti-angiogenic effect of caffeic acid on retinal neovascularization. Vasc Pharmacol 51: 262-267.

83. Kowluru RA, Odenbach S (2004) Effect of Long-Term Administration of alphaLipoic Acid on Retinal Capillary Cell Death and the Development of Retinopathy in Diabetic Rats. Diabetes 53: 3233-3238.

84. Petersena M, Simmonds MSJ (2003) Rosmarinic acid. Phytochemistry 62 $121-125$

85. Kern TS, Tang J, Mizutani M, Kowluru RA, Nagaraj RH, et al. (2000) Response of capillary cell death to aminoguanidine predicts the development of retinopathy: comparison of diabetes and galactosemia. Invest Ophthalmol Vis Sci 41: 3972-3978.

86. Biegelsen ES, Vita JA (1999) Human studies of antioxidants and vascula function. In: Keaney Jr. JF, editor. Oxidative stress and vascular disease. Dordrecht: Kluwer Academic Publishers: 213-243.

87. Gaziano JM (1999) Antioxidants and cardiovascular disease. In: Keaney Jr JF, editor. Oxidative stress and vascular disease. Dordrecht: Kluwer Academic Publishers: 245-258.
88. Hammes HP, Du X, Edelstein D, Taguchi T, Matsumura $T$, et al. (2003) Benfotiamine blocks three major pathways of hyperglycemic damage and prevents experimental diabetic retinopathy. Nat Med 9: 294-299.

89. Moustafa SA (2004) Zinc might protect oxidative changes in the retina and pancreas at the early stage of diabetic rats. Toxicol Appl Pharmacol 20: 149155

90. McCarty MF (2005) The putative therapeutic value of high-dose selenium in proliferative retinopathies may reflect downregulation of VEGF production by the hypoxic retina. Med Hypotheses 64: 159-161.

91. Mandal NA, Patlolla JMR, Zheng L, Agbaga MP, Tran JA, (2009) Curcumin protects retinal cells from light-and oxidant stress-induced cell death. Free Radical Biol Med 46 :672-679.

92. Spadea L, Balestrazzi E (2001) Treatment of vascular retinopathies with Pycnogenol. Phytother Res 15: 219-223.

93. Di Leo MA, Ghirlanda G, Gentiloni Silveri N, Giardina B, Franconi F, et al (2003) Potential therapeutic effect of antioxidants in experimental retina: a comparison between taurine and vitamin $\mathrm{E}$ plus selenium supplementation. Free Radical Res 37: 323-330.

94. Banday AA, Marwaha A, Tallam LS, Lokhandwala MF (2005) Tempol Reduces Oxidative Stress, Improves Insulin Sensitivity, Decreases Renal Dopamine D1 Receptor Hyperphosphorylation, and Restores D1 Receptor-G-Protein Coupling and Function in Obese Zucker Rats. Diabetes 54: 2219-2226.

95. Sabu MC, Smitha K, Kuttan R (2002) Anti-diabetic activity of green tea polyphenols and their role in reducing oxidative stress in experimental diabetes. J Ethnopharmacol 83: 109-116.

96. Vinson JA, Zhang J (2005) Black and green teas equally inhibit diabetic cataracts in a streptozotocin-induced rat model of diabetes. J Agric Food Chem 53: $3710-3713$

97. Sherris D (2007) Ocular drug development - Future Directions. Angiogenesis 10: $71-76$.

98. Penn JS, Madan A, Caldwell RB, Bartoli M, Caldwell RW, et al. (2008) Vascular endothelial growth factor in eye disease. Prog Retin Eye Res 27: 331- 371.

99. Anderson OA, Bainbridge JW, Shima DT (2010) Delivery of anti-angiogenic molecular therapies for retinal disease. Drug Discov Today 15: 272-282.

100. Mohamed Q, Wong TY (2008) Emerging drugs for diabetic retinopathy. Expet Opin Emerg Drugs 13(4):675-94

101. Millen AE, Klein R, Folsom AR, Stevens J, Palta M, et al. (2004) Relation between intake of vitamins $C$ and $E$ and risk of diabetic retinopathy in the Atherosclerosis Risk in Communities Study. Am J Clin Nutr 79: 865-873. 